\title{
CONSTRUÇÃO E VALIDAÇÃO DE INSTRUMENTO PARA AVALIAR CONHECIMENTOS DE UNIVERSITÁRIOS SOBRE TRANSTORNO ESPECÍFICO DA APRENDIZAGEM
}

Camila Barbosa Riccardi León

Universidade Presbiteriana Mackenzie

Ronê Paiano

Universidade Presbiteriana Mackenzie

Analice Oliveira Fragoso

Universidade Presbiteriana Mackenzie

\section{Alisson Rogério Caetano de Siqueira}

Universidade Presbiteriana Mackenzie

Maria Cristina Triguero Veloz Teixeira

Universidade Presbiteriana Mackenzie

\author{
Alessandra Gotuzo Seabra \\ Universidade Presbiteriana Mackenzie
}

\section{Resumo}

O Transtorno Específico da Aprendizagem (TEAp) caracteriza-se por dificuldades persistentes para desenvolver habilidades acadêmicas de leitura, escrita e/ou matemática, sendo fundamental que professores conheçam suas características. 0 estudo teve como objetivos construir um questionário que avalia conhecimentos sobre TEAp e verificar suas propriedades psicométricas, mediante estudo piloto com estudantes de pedagogia ingressantes e concluintes. Participaram 178 estudantes, entre 18 e 54 anos, de três instituições de ensino superior particulares. Resultados revelaram índice adequado de consistência interna (Alfa de Cronbach $=0,738$ ). Houve maior conhecimento sobre TEAp dos estudantes concluintes em relação aos ingressantes, porém com resultados baixos em alguns itens. Também houve diferença significativa entre as instituições. Não houve associação dos fatores idade, ano, renda e situação de trabalho, com o nível de conhecimento. Além de contribuir com a criação do questionário, os resultados sinalizam a necessidade de incluir disciplinas sobre TEAp na grade curricular dos cursos de pedagogia.

Palavras-chave: pedagogia; dislexia; questionário; educação; distúrbios da aprendizagem. 


\title{
CONSTRUCTION AND VALIDATION OF A QUESTIONNAIRE TO ASSESS PEDAGOGY STUDENTS' KNOWLEDGE ON SPECIFIC LEARNING DISORDER
}

\begin{abstract}
Specific Learning Disorder (SLD) is characterized by persistent difficulties in developing reading, writing, and math. It's essential that teachers know their characteristics. The study aimed to develop a questionnaire to assess knowledge on SLD and to verify its psychometric properties. It was conducted a pilot study involving incoming and graduating students of pedagogy courses. Participated 178 students aged between 18 and 54, from 3 private higher education institutions. The results revealed adequate indexes of internal consistency (Cronbach's alpha $=0.738$ ). Graduating students showed to have more knowledge than incoming students did, but with low results on some items. There was also a significant difference between institutions. There was no association of others factors, such as age, year, income, and work situation, with the students' level of knowledge. Besides contributing to the questionnaire's development, the results indicate the need to include courses on SLD in the curriculum of the pedagogy programs.
\end{abstract}

Keywords: pedagogy; dyslexia; questionnaire; education; learning disabilities.

\section{CONSTRUCCIÓN Y VALIDACIÓN DE INSTRUMENTO PARA LA EVALUACIÓN DE CONOCIMIENTOS DE ESTUDIANTES DE PEDAGOGÍA SOBRE EL TRASTORNO ESPECÍFICO DEL APRENDIZAJE}

\begin{abstract}
Resumen
El Trastorno Específico del Aprendizaje (TEAp) se caracteriza por dificultades persistentes para desarrollar lectura, escritura y matemática, siendo fundamental que profesores conozcan sus características. El estudio tuvo como objetivos elaborar un cuestionario que evalúa conocimientos sobre el TEAp y verificar sus propiedades psicométricas. Participaron del estudio piloto 178 estudiantes de pedagogía ingresantes y concluyentes, entre 18 y 54 años, de tres instituciones privadas de enseñanza universitaria. Los resultados revelaron índice adecuado de consistencia interna (Alfa de Cronbach $=0,738$ ). Hubo mayor conocimiento sobre TEAp en los concluyentes en relación a los ingresantes, sin embargo, con resultados bajos en algunos ítems. También hubo diferencia significativa entre las instituciones. No hubo asociación de otros factores investigados con el nivel de conocimiento. Además de contribuir con la creación del cuestionario, los resultados señalizan la necesidad de que sea incluida asignaturas sobre el TEAp en los planes de estudios de licenciaturas en pedagogía.
\end{abstract}

Palabras clave: pedagogía; dislexia; cuestionario; educación; trastornos de aprendizaje.

\section{INTRODUÇÃO}

O Transtorno Específico da Aprendizagem (TEAp), também conhecido como Distúrbio de Aprendizagem ou Transtorno de Aprendizagem, tem origem neurobiológica e caracteriza-se por dificuldades persistentes (não transitórias) para desenvolver habilidades acadêmicas de leitura, escrita e/ou matemática. 0 transtorno acomete entre 5 e 15\% de crianças em idade escolar, em diferentes idiomas e culturas (American Psychiatric Association [APA], 2014). É importante destacar que não são caracterizados como TEAp os casos em que tais habilidades acadêmicas encontram-se prejudicadas em função de outras condições clínicas, condições transitórias, absenteísmo e/ou fatores ambientais ligados à estimulação social, educacional e pedagógica (Flatters, Hill, Williams, Barber, \& Mon-Williams, 2014; Osborn \& Pereira, 2012; Silva \& Cavalcante, 2015). 
O TEAp pode ser classificado de acordo com as habilidades que se encontram prejudicadas. A classificação mais atual, descrita pelo Manual Diagnóstico e Estatístico de Transtorno Mentais/ DSM-5 (APA, 2014), especifica os prejuízos em termos de domínios e sub-habilidades acadêmicas. Em relação aos domínios, o TEAp pode ser classificado como: TEAp com prejuízo na leitura (problemas na precisão, fluência e/ou compreensão da leitura), TEAp com prejuízo na expressão escrita (problemas na precisão da ortografia, da gramática e/ou da pontuação; déficits na clareza ou organização da expressão escrita) e TEAp com prejuízo na matemática (prejuízos no senso numérico, na memorização de fatos aritméticos, nos cálculos e/ou no raciocínio matemático). A dislexia é um termo alternativo para prejuízos na leitura, com exceção de déficits em compreensão, e a discalculia é um termo alternativo para prejuízos na matemática, com exceção de déficits no raciocínio matemático. Além, segundo o DSM-5 (APA, 2014), é possível classificar a intensidade da manifestação dos graus em leve, moderado e grave.

A etiologia do TEAp é multifatorial e seu diagnóstico é clínico; portanto, sua avaliação deve ser multidisciplinar, a fim de excluir outras possíveis causas das dificuldades acadêmicas manifestadas, como problemas sensoriais, deficiência intelectual, instrução educacional inadequada, dentre outros. A avaliação multidisciplinar contribui também para diagnóstico diferencial, uma vez que é comum haver comorbidade com outros transtornos, principalmente com o Transtorno de Déficit de Atenção/ Hiperatividade-Impulsividade - TDAH (APA, 2014).

Geralmente o diagnóstico tende a ocorrer após o início da educação formal, quando as demandas acadêmicas aumentam (APA, 2014). Nesse sentido, os professores têm papel importante na observação e acompanhamento do desempenho acadêmico dos alunos e podem ser os primeiros a identificar precocemente sinais compatíveis com perfil de TEAp (Berninger, Nagy, Richards, \& Raskind, 2008; Padhy et al., 2015).

Dois fatores podem ser citados como exemplos para entender as dificuldades vivenciadas por um professor ao levantar uma suspeita de TEAp. De um lado, trata-se de um transtorno complexo frequentemente sobreposto com outras comorbidades psiquiátricas (de Wit et al., 2018). De outro, em países em desenvolvimento, como é caso do Brasil, continua prevalecendo desconhecimento por parte do professor sobre instrumentos adequados de avaliação de sinais de TEAp, o que também interfere na identificação precoce do transtorno nos primeiros anos de vida escolar (Fortes et al., 2016).

Para o levantamento de suspeita de um TEAp, os professores precisam perceber que as dificuldades que o aluno apresenta para desenvolver habilidades acadêmicas básicas estão interferindo ou até impedindo a aprendizagem de outras matérias acadêmicas, configurando muitas vezes a persistência dos sinais. Usualmente, o professor é quem fará os devidos encaminhamentos desse aluno, 
o que é fundamental para compreender melhor o quadro e descartar outras condições do neurodesenvolvimento que também afetam a aprendizagem escolar, mas são distintas do TEAp, por exemplo, deficiência intelectual, atraso global do desenvolvimento, deficiências auditivas ou visuais, problemas neurológicos ou motores, dentre outros (APA, 2014). Em função disso, é importante investigar o nível de conhecimento que professores têm sobre TEAp para verificar se, de fato, eles conseguem identificar sinais e características de um perfil compatível com TEAp.

Alguns estudos em países em desenvolvimento como a Índia (Kamala \& Ramganesh, 2013; Lingeswaran, 2013; Padhy et al., 2015) têm explorado a percepção que professores da Educação Básica têm sobre o TEAp. Por exemplo, Padhy et al. (2015) avaliaram conhecimentos sobre o transtorno em 80 professores de $3^{a}$ e $4^{a}$ séries (atualmente $4^{\circ}$ e $5^{\circ}$ ano) do ensino fundamental de escolas públicas urbanas, rurais e em favelas de Chandigarh, na Índia. A amostra foi randomizada a partir de 103 escolas, das quais 20 escolas foram selecionadas. Verificou-se que, apesar de a maioria dos participantes ter formação acadêmica adequada (48,8\% com pós-graduação e $47,5 \%$ com graduação), experiência profissional na área (57,5\% acima de 5 anos) e experiência prévia de alunos com TEAp na escola (67,5\%), um número expressivo de professores $(43,7 \%)$ declarou não ter conhecimento sobre o TEAp. Os dados revelam que a falta de conhecimento do professor sobre o assunto contribui para que alunos com TEAp não sejam precocemente identificados no ambiente de sala de aula, atrasando a avaliação diagnóstica e, consequentemente, dificultando o acesso a serviços e atendimentos educacionais específicos para desenvolvimento de habilidades de leitura, escrita ou matemática (Berninger et al., 2008).

A percepção sobre TEAp dos professores da Educação Básica parece variar de acordo com o tempo de experiência e aspectos culturais. Estudos conduzidos nos Estados Unidos e Reino Unido (Washburn, Cantrell, \& Joshi, 2013) e na Espanha e Peru (Soriano-Ferrer, Echegaray-Bengoa, \& Joshi, 2016) identificaram que professores, em comparação aos estagiários, tendem a reconhecer melhor a condição sintomática e diagnóstica da dislexia, apresentando apenas alguns equívocos comuns.

Ainda que escassos, os estudos focados na avaliação de conhecimentos sobre TEAp em professores (Kamala \& Ramganesh, 2013; Lingeswaran, 2013; Padhy et al., 2015; Soriano-Ferrer et al., 2016; Washburn et al., 2013) corroboram a importância de que futuros professores da educação básica, que muitas vezes já atuam nas escolas como estagiários, tenham disciplinas curriculares no curso de graduação que contemple as características, principais sinais e sintomas do TEAp, bem como fatores associados e condições comórbidas. Assim, poderão estar mais bem preparados para identificar 
dificuldades acadêmicas que vão além do que seria esperado para a série e a idade de seus alunos.

No contexto nacional a temática parece ser pouco estudada, sendo mais recente o interesse nos últimos anos, conforme revelado em estudos anteriores (Carvalho, Crenitte, \& Ciasca, 2007; Dias, Pereira, \& Van Borsel, 2013; Gonçalves \& Crenitte, 2014; Lopes \& Crenitte, 2013; Machado, Borges, Costa, \& Almeida, 2015; Pereira, Simões, Siqueira, \& Alves, 2011; Tratis \& Gomes, 2009). Tais pesquisas foram realizadas apenas com professores do ensino fundamental e utilizaram diferentes instrumentos de avaliação do conhecimento do professor acerca das características e critérios diagnósticos do TEAp.

Dentre essas pesquisas, três (Machado et al., 2015; Pereira et al., 2011; Tratis \& Gomes, 2009) não disponibilizaram o questionário utilizado, duas (Dias et al., 2013; Lopes \& Crenitte, 2013) utilizaram questionários com 18 questões, uma com 15 questões (Gonçalves \& Crenitte, 2014) e uma com 3 questões (Carvalho et al., 2007). Quanto aos instrumentos utilizados, duas pesquisas adaptaram instrumentos de estudos anteriores, sendo que o trabalho de Gonçalves e Crenitte (2014) utilizou o questionário elaborado por Gonçalves (2011) e o trabalho de Lopes e Crenitte (2013) adaptou o questionário de Fernandes e Crenite (2008). Nos demais estudos (Carvalho et al., 2007; Dias et al., 2013; Machado et al., 2015; Pereira et al., 2011; Tratis \& Gomes, 2009), os questionários foram elaborados pelos próprios autores e nem sempre há descrição das propriedades psicométricas do instrumento. Outra limitação desses estudos é que a maioria focaliza a dislexia ou a diferenciação entre TEAp e dificuldade de aprendizagem, sendo que apenas um estudo (Dias et al., 2013) abordou questões relativas à discalculia. Adicionalmente, ainda dentre os estudos, apenas três citam como referencial teórico um manual de classificação diagnóstica internacional, como o DSM-versão IV ou CID-10 (Gonçalves \& Crenitte, 2014; Machado et al., 2015; Pereira et al., 2011).

Pesquisas brasileiras têm apontado que a temática do TEAp precisa ser mais estudada em relação à formação dos professores, já que muitos deles são obrigados a procurar formação extracurricular para estudar o assunto (Carvalho et al., 2007; Dias et al., 2013; Gonçalves \& Crenitte, 2014; Lopes \& Crenitte, 2013; Machado et al., 2015; Pereira et al., 2011; Tratis \& Gomes, 2009). Apesar desse panorama, o trabalho de Pereira e colaboradores (2011) revelou resultados relativamente positivos quando explorou, em um grupo de professores, o nível de conhecimento sobre a dislexia, identificando que aproximadamente $75 \%$ da amostra apresentava conhecimentos adequados sobre - quadro clínico, suas principais características, causas e capacidade de reconhecimento dos profissionais envolvidos em todo processo. Já os demais estudos (e.g., Carvalho et al., 2007; Dias et al., 2013; Gonçalves \& Crenitte, 2014; Lopes \& Crenitte, 2013; Machado et al., 2015; Tratis \& Gomes, 2009) verificaram que os professores não têm domínio suficiente sobre as 
características de um TEAp, sentindo-se inseguros para lidar com crianças que apresentam tais características em sala de aula.

No Brasil não há, até o momento, evidências de estudos com outro público, para além dos professores formados, ou estudos que verifiquem se, no decorrer do curso de pedagogia, outros fatores estão associados com o nível de conhecimentos dos estudantes sobre TEAp. De outro lado, tampouco têm sido divulgados estudos sobre construção e verificação de propriedades psicométricas de instrumentos que avaliem conhecimentos sobre TEAp em estudantes de cursos de pedagogia.

Considerando a importância do futuro professor como principal agente na identificação precoce de alunos com risco para TEAp, os objetivos do estudo foram construir um questionário que avalia conhecimentos sobre TEAp e verificar suas propriedades psicométricas mediante estudo piloto junto a estudantes de pedagogia ingressantes e concluintes. Foram explorados, também, o efeito dos fatores idade, ano, renda, número de horas de trabalho e instituição sobre o nível de conhecimentos sobre TEAp. Diferentemente das pesquisas identificadas pela revisão teórica realizada, a originalidade deste estudo reside na amostra composta por estudantes de pedagogia e na utilização do DSM-5 como referencial teórico para a elaboração do questionário.

\section{MÉTODO}

\section{Participantes}

O desenho do estudo foi transversal com amostra formada por 178 graduandos de cursos de pedagogia (amostra por conveniência), entre 18 a 54 anos (idade média $=34$ anos; $D P=25,46$ ), de ambos os sexos, de três Instituições de Ensino Superior (IES) particulares, sendo duas localizadas no estado de São Paulo e uma no Paraná. Foram considerados como ingressantes os alunos regularmente matriculados no $1^{\circ}$ ou $2^{\circ}$ semestre e, como concluintes, os matriculados entre $5^{\circ}$ e $8^{\circ}$ semestres. Foi pré-requisito para os ingressantes do $1^{0}$ ano não terem cursado nenhuma disciplina com conteúdo relacionado ao TEAp. Assim, dos 345 alunos matriculados nas três instituições, apenas 178 preencheram os critérios de inclusão, conforme apresentado na Tabela 1.

Tabela 1.

Caracterização da amostra final por instituição, ano e idade.

\begin{tabular}{ccccccc}
\hline \multirow{2}{*}{ Instituição } & \multirow{2}{*}{ Ano } & Número de & \multicolumn{4}{c}{ Idade } \\
\cline { 4 - 7 } & alunos & Min. & Máx. & Média & DP \\
\hline \multirow{3}{*}{1} & Ingressantes & 13 & 18 & 38 & 28 & 14,14 \\
& Concluintes & 19 & 22 & 46 & 34 & 16,97 \\
& Subtotal & 32 & 18 & 46 & 32 & 19,80 \\
\cline { 4 - 7 }
\end{tabular}




\begin{tabular}{ccccccc}
\cline { 3 - 7 } 2 & Ingressantes & 38 & 18 & 54 & 36 & 25,46 \\
& Concluintes & 12 & 20 & 53 & 36,5 & 23,33 \\
Subtotal & 50 & 18 & 54 & 36 & 25,46 \\
\hline \multirow{3}{*}{3} & Ingressantes & 52 & 18 & 47 & 32,5 & 20,51 \\
& Concluintes & 44 & 20 & 51 & 35,5 & 21,92 \\
$\quad$ Subtotal & 96 & 18 & 54 & 36 & 25,46 \\
\hline
\end{tabular}

\section{Instrumento}

O questionário estruturado foi elaborado pelos autores, baseado nos critérios diagnósticos do DSM-5 (APA, 2014), para identificação de características e critérios diagnósticos do TEAp. O questionário foi disponibilizado na íntegra em $<$ https://goo.gl/forms/tDbtUafyy2c5kUn52>. Como observado, o instrumento foi dividido em duas partes. Na primeira parte, são averiguados dados sociodemográficos, como renda (medida em termos de salários mínimos), situação de trabalho em termos de vínculos empregatícios ou de estágio (medido em termos de número de horas semanais), cursos prévios sobre TEAp, formação, idade, semestre/ instituição), bem como conhecimentos gerais sobre definição de TEAp. Na segunda parte, o questionário é composto por 20 afirmações sobre as características e critérios diagnósticos do TEAp para assinalar como 'verdadeiro', 'falso' ou 'não sei'.

Para se chegar à versão final da segunda parte do questionário foram realizadas três etapas: 1) elaboração pelos autores de afirmações acerca das características e critérios diagnósticos do TEAp, conforme descrito no DSM-5 (APA, 2014), o que resultou em 24 itens; 2) redução e reescrita para 20 afirmações após análise interna pelos autores, visando à união de questões cujos conteúdos se sobrepunham e à aproximação da quantidade de questões de outros instrumentos existentes (e.g. Dias et al., 2013; Lopes \& Crenitte, 2013); 3) condução de piloto em 10 alunas de graduação de uma IES que não participaram do estudo principal e em 2 pedagogas formadas com pós-graduação em psicopedagogia, para verificar o tempo de aplicação, a compreensão das afirmações e de possíveis dúvidas.

No questionário, a correção da parte 1 foi realizada por meio de transcrição literal para as questões abertas e pela tabulação das variáveis para as questões fechadas. Para a correção da parte 2, foi adotado o critério de acertos e erros, sendo utilizada a pontuação de 0 para erros e 1 para acertos, com base nos critérios diagnósticos descritos pelo DSM-5 (APA, 2014). Respostas marcadas como 'não sei' também foram tabuladas como 0. 


\section{Procedimentos}

O projeto foi aprovado pelo Comitê de Ética em Pesquisa (CAAE no. 56235015.7.0000.0084). O questionário foi desenvolvido pelos autores do artigo, conforme descrito anteriormente.

Para aplicação do questionário, foi inicialmente realizado o contato com as instituições que autorizam a realização da pesquisa. Em seguida, as coletas foram agendadas. Nas instituições de São Paulo, foi acordado que, após a coleta, fosse realizada uma palestra explicativa sobre as características e os critérios diagnósticos do TEAp, com duração de uma hora. A instituição do Paraná não manifestou interesse na palestra. O tempo de aplicação do questionário foi de aproximadamente 20 minutos e a mesma ocorreu na própria sala de aula dos graduandos, com autorização de seus professores.

Foram realizadas análises estatísticas descritivas e inferenciais por meio do programa Statistical Package for the Social Sciences (SPSS), versão 22.0. Para analisar a precisão do questionário, foi calculado o alfa de Cronbach e foi realizada análise dos itens. Para buscar evidências de validade por relação com outras variáveis, foi verificado se houve diferença no conhecimento sobre o TEAp entre alunos ingressantes e concluintes pelo teste de Kruskal-Wallis. De forma exploratória, foram também testadas associações entre o conhecimento dos alunos sobre TEAp e as variáveis instituição (por meio de análise do teste de Kruskal-Wallis), idade, ano, renda e situação de trabalho (por meio de correlação de Spearman). Ressalta-se que foram conduzidas análises não-paramétricas devido à distribuição não-normal dos dados.

\section{RESULTADOS}

Inicialmente, foram calculadas as estatísticas descritivas relativas a média e desvio padrão de cada item para amostra total e separadamente por momento (ingressantes e concluintes). Tais resultados estão apresentados na Tabela 2 ( $n$ total $=178, \mathrm{n}$ ingressantes $=103, \mathrm{n}$ concluintes $=75$ ). Observou-se que, na amostra total, a maior média de acertos foi de 0,72 , no item 4 (item: "O TA se constitui, simplesmente, por uma consequência de falta de oportunidade de aprendizagem ou educação escolar inadequada"). Por outro lado, as médias mais baixas $(0,07)$ foram observadas nos itens 18 ("O TA é mais comum no sexo masculino do que no feminino") e 20 ("O TDAH é considerado um dos transtornos específicos da aprendizagem"). Também foram baixas $(0,13)$ as médias encontradas nos itens 5 ("As dificuldades de aprendizagem oriundas de um TA podem ter início tanto durante os anos escolares, como na fase adulta"), 6 ("As dificuldades de aprendizagem oriundas de um TA podem ser explicadas a partir do diagnóstico de deficiências intelectuais, acuidade visual ou auditiva não corrigida, outros transtornos mentais ou neurológicos") e 17 ("O risco relativo de 
TA é substancialmente maior em parentes de $1^{\circ} \mathrm{grau}$ de indivíduos com essas dificuldades").

Analisando-se separadamente ingressantes e concluintes, observa-se que a maior média de acertos $(0,65)$ para os ingressantes foi no item 3 ("Uma característica essencial do TA são dificuldades persistentes para aprender habilidades acadêmicas fundamentais, tais como leitura, ortografia, escrita e/ou matemática") e, para os concluintes, com média de 0,88 , foi o item 4 (descrito anteriormente). A menor média de acertos $(0,07)$ para ingressantes foi no item 2 ("O TA não tem origem neurobiológica") e para concluintes foi no item 18 ("O TA é mais comum no sexo masculino do que no feminino"), com média de acertos de 0,05 .

Tabela 2.

Distribuição de médias e desvio padrão (DP) de número de acertos por itens entre os grupos de estudantes ingressantes e concluintes.

\begin{tabular}{cccc}
\hline Item & $\begin{array}{c}\text { Média e DP } \\
\text { total }\end{array}$ & $\begin{array}{c}\text { Média e DP - } \\
\text { ingressantes }\end{array}$ & $\begin{array}{c}\text { Média e DP - } \\
\text { concluintes }\end{array}$ \\
\hline 1 & $0,59(0,49)$ & $0,54(0,50)$ & $0,65(0,48)$ \\
2 & $0,28(0,45)$ & $0,25(0,44)$ & $0,32(0,47)$ \\
3 & $0,66(0,48)$ & $0,65(0,48)$ & $0,67(0,47)$ \\
4 & $0,72(1,11)$ & $0,61(0,49)$ & $0,88(1,60)$ \\
5 & $0,13(0,34)$ & $0,08(0,27)$ & $0,20(0,40)$ \\
6 & $0,13(0,34)$ & $0,14(0,34)$ & $0,12(0,33)$ \\
7 & $0,61(0,49)$ & $0,50(0,50)$ & $0,76(0,43)$ \\
8 & $0,31(0,46)$ & $0,28(0,45)$ & $0,35(0,48)$ \\
9 & $0,15(0,36)$ & $0,11(0,31)$ & $0,21(0,41)$ \\
10 & $0,35(0,48)$ & $0,25(0,44)$ & $0,49(0,50)$ \\
11 & $0,44(0,50)$ & $0,37(0,48)$ & $0,55(0,50)$ \\
12 & $0,25(0,43)$ & $0,21(0,41)$ & $0,29(0,46)$ \\
13 & $0,51(0,50)$ & $0,48(0,50)$ & $0,56(0,50)$ \\
14 & $0,42(0,49)$ & $0,36(0,48)$ & $0,51(0,50)$ \\
15 & $0,43(0,50)$ & $0,42(0,50)$ & $0,45(0,50)$ \\
16 & $0,30(0,46)$ & $0,22(0,42)$ & $0,41(0,50)$ \\
17 & $0,13(0,34)$ & $0,11(0,31)$ & $0,16(0,37)$ \\
18 & $0,07(0,26)$ & $0,09(0,28)$ & $0,05(0,23)$ \\
19 & $0,26(0,44)$ & $0,24(0,43)$ & $0,28(0,45)$ \\
20 & $0,07(0,25)$ & $0,07(0,25)$ & $0,07(0,25)$ \\
\hline & & & \\
\hline
\end{tabular}

Em seguida, foi conduzida análise de consistência interna do instrumento. O alfa de Cronbach resultante foi de 0,738, um valor considerado aceitável (George \& Mallery, 2003). Os resultados da análise de itens podem ser observados na Tabela 3, que sumariza dados de média de escore da escala se o 
item for deletado, variância da escala se o item for deletado, correlação itemtotal e alfa de Cronbach se o item for deletado. Observa-se que o alfa de Cronbach aumentaria se o item 20 fosse deletado, item que apresentou a menor correlação com o escore total do questionário.

Tabela 3.

Estatísticas relativas à análise de precisão.

\begin{tabular}{ccccc}
\hline Item & $\begin{array}{c}\text { Média da escala se } \\
\text { o item for } \\
\text { deletado }\end{array}$ & $\begin{array}{c}\text { Variância se o } \\
\text { item for } \\
\text { deletado }\end{array}$ & $\begin{array}{c}\text { Correlação item- } \\
\text { total }\end{array}$ & $\begin{array}{c}\text { Alfa de Cronbach } \\
\text { se } \\
\text { o item for } \\
\text { deletado }\end{array}$ \\
\hline 1 & 6,24 & 14,114 & 0,434 & 0,717 \\
2 & 6,54 & 14,408 & 0,395 & 0,721 \\
3 & 6,17 & 13,960 & 0,500 & 0,712 \\
4 & 6,10 & 13,493 & 0,153 & 0,779 \\
5 & 6,70 & 15,331 & 0,197 & 0,735 \\
6 & 6,70 & 15,399 & 0,171 & 0,736 \\
7 & 6,21 & 14,078 & 0,449 & 0,716 \\
8 & 6,52 & 14,669 & 0,304 & 0,728 \\
9 & 6,67 & 15,419 & 0,147 & 0,738 \\
10 & 6,47 & 14,149 & 0,439 & 0,717 \\
11 & 6,38 & 14,430 & 0,340 & 0,725 \\
12 & 6,58 & 14,449 & 0,404 & 0,721 \\
13 & 6,31 & 13,923 & 0,478 & 0,713 \\
14 & 6,40 & 14,163 & 0,417 & 0,718 \\
15 & 6,39 & 14,330 & 0,369 & 0,722 \\
16 & 6,52 & 14,533 & 0,346 & 0,725 \\
17 & 6,70 & 15,297 & 0,210 & 0,734 \\
18 & 6,75 & 15,487 & 0,199 & 0,735 \\
19 & 6,57 & 14,654 & 0,332 & 0,726 \\
20 & 6,76 & 15,834 & 0,033 & \\
\hline
\end{tabular}

Para buscar evidências de validade por relação com outras variáveis, verificou-se se houve diferença no conhecimento sobre o TEAp entre alunos ingressantes e concluintes. Como a distribuição mostrou-se não-normal, conforme o resultado do teste de normalidade Kolmogorov-Smirnov $(p=0,015)$, foram realizadas análises descritivas e inferenciais não-paramétricas do momento escolar (alunos ingressantes e concluintes) sobre o escore total de acertos no questionário aplicado. A Tabela 4 sumariza as estatísticas descritivas. Os resultados sugerem que os alunos concluintes tenderam a apresentar maior conhecimento do que os ingressantes, medido pelo total de acertos (média e 
mediana). De fato, a diferença significativa entre grupos (ingressantes e concluintes) foi confirmada pelo teste de Kruskal-Wallis $(p=0,003)$.

Tabela 4.

Estatísticas descritivas do número de acertos no questionário em função do momento escolar (alunos ingressantes e concluintes) e do tipo de instituição.

\begin{tabular}{|c|c|c|c|c|c|c|c|}
\hline \multirow[b]{2}{*}{ Momento escolar } & \multirow[b]{2}{*}{$\begin{array}{c}\text { Média } \\
\text { de } \\
\text { acertos }\end{array}$} & \multirow[b]{2}{*}{$\begin{array}{l}\text { Mediana } \\
\text { de } \\
\text { acertos }\end{array}$} & \multicolumn{2}{|c|}{$\begin{array}{c}\text { Intervalo de } \\
\text { confiança } 95 \%\end{array}$} & \multirow[b]{2}{*}{ DP } & \multirow[b]{2}{*}{ Mín. } & \multirow[b]{2}{*}{ Máx. } \\
\hline & & & $\begin{array}{l}\text { Limite } \\
\text { inferior }\end{array}$ & $\begin{array}{l}\text { Limite } \\
\text { superior }\end{array}$ & & & \\
\hline Ingressantes & 5,98 & 6,00 & 5,17 & 6,79 & 4,15 & 0 & 16 \\
\hline Concluintes & 7,80 & 8,00 & 7,08 & 8,52 & 3,12 & 0 & 13 \\
\hline \multicolumn{8}{|l|}{ Instituição } \\
\hline 1 & 6,09 & 5,50 & 5,05 & 7,14 & 2,88 & 0 & 11 \\
\hline 2 & 7,14 & 8,00 & 5,96 & 8,32 & 4,14 & 0 & 14 \\
\hline 3 & 6,76 & 7,00 & 5,95 & 7,57 & 3,97 & 0 & 16 \\
\hline
\end{tabular}

Posteriormente, de forma exploratória, verificou-se se houve diferenças no conhecimento sobre o TEAp, medido pelo número total de acertos, entre as três instituições. Para tanto, foram conduzidas análises descritivas e inferenciais pelo teste de Kruskal-Wallis, conforme também descrito na Tabela 4. Em termos descritivos, as médias de acerto entre as instituições foram próximas (variando de 6,09 a 7,14), sendo que a instituição 2, localizada em São Paulo, tendeu a ter média de acertos maior do que as demais instituições. Apesar de próximas, o teste de Kruskal-Wallis identificou diferença significativa entre grupos ( $p=$ 0,033), revelando diferença significativa no conhecimento sobre o TEAp, medido pelo escore total de acertos, em diferentes instituições.

Finalmente, também de forma exploratória, foi conduzida a análise de correlação de Spearman para analisar quais fatores, entre idade, ano, renda e situação de trabalho (em termos de número de horas trabalhadas por semana), se correlacionaram com o conhecimento que os alunos têm sobre TEAp, conforme ilustra a Tabela 5.

Tabela 5.

Associação entre total de acertos no questionário com idade, ano, renda e situação de trabalho mediante coeficiente de correlação de Spearman.

\begin{tabular}{lccccc}
\hline & & Idade & Ano & Renda & $\begin{array}{c}\text { Situação de } \\
\text { trabalho }\end{array}$ \\
\hline Total de & rho & 0,143 & $0,225^{* *}$ & 0,023 & 0,025 \\
acertos & $p$ & 0,057 & 0,003 & 0,762 & 0,744 \\
& $\mathrm{~N}$ & 178 & 178 & 178 & 178 \\
\hline
\end{tabular}


**A correlação é significativa no nível 0,01 .

A análise de correlação de Spearman mostrou apenas um coeficiente de correlação estatisticamente significativo, positivo, entre o ano que o participante estava cursando e o número de acertos. Porém, o coeficiente foi baixo. Assim, o ano em que o aluno está matriculado parece se relacionar com o conhecimento de TEAp, medido pelo total de acertos, porém de forma baixa, o que corrobora a diferença observada entre ingressantes e concluintes.

\section{DISCUSSÃO}

Inicialmente, foram verificados os indicadores de precisão e analisados os itens do questionário, os quais ambos revelaram índices adequados. O Alfa de Cronbach de 0,738 mostrou-se adequado, revelando boa precisão do questionário. A análise de itens relevou que apenas a exclusão do item 20 aumentaria o alfa, porém os autores consideraram o item claro e objetivo, de forma que esse dado relativo ao aumento de precisão com a sua exclusão é provavelmente devido mais à ausência de conhecimento dos participantes acerca de seu conteúdo, do que um problema intrínseco ao item.

Posteriormente, buscaram-se evidências de validade por relação com outras variáveis por meio das diferenças nos resultados entre alunos ingressantes e concluintes de cursos de pedagogia. Tais análises demonstraram que o conhecimento dos concluintes de pedagogia dos três cursos foi significativamente maior do que o conhecimento dos ingressantes, medido pelo total de acertos no questionário. Por exemplo, analisando os cinco itens do questionário que tiveram maior percentual de acertos, ingressantes e concluintes tenderam a acertar os mesmos itens, a saber, itens 1, 3, 4, 7 e 13. Tais itens referem-se mais a aspectos gerais do TEAp, como características, definição, compreensão da condição neurobiológica e diagnóstico. Porém, a média de acertos dos concluintes nestes itens foi maior $(70,4 \%)$ do que dos ingressantes (55,6\%). Pode-se citar, por exemplo, o item 7 "Dislexia é um TA com prejuízo nas habilidades de leitura como: problemas no reconhecimento preciso ou fluente de palavras, problemas de decodificação", que teve $76 \%$ de acerto entre os concluintes e $50 \%$ entre os ingressantes. Talvez este resultado possa estar associado não só ao fato de terem sido expostos a maior quantidade conhecimentos sobre o transtorno, mas também ao tempo de experiência docente, considerando que alunos concluintes em pedagogia possam ter mais tempo de experiência em sala de aula do que os alunos ingressantes. De fato, estudos anteriores conduzidos em outros países (Soriano-Ferrer et al., 2016; Washburn et al., 2013) têm revelado que o tempo de experiência docente pode impactar na percepção sobre TEAp, ou seja, que professores tendem a 
reconhecer melhor os sinais de dislexia nos alunos, em comparação aos estagiários.

Por outro lado, dos cinco itens do questionário que tiveram menor percentual de acertos, quatro foram os mesmos entre ingressantes e concluintes (itens 5, 17, 18 e 20). Tais itens referem-se a aspectos específicos do TEAp, como critérios diagnósticos, risco relativo e prevalência. Tal resultado corrobora estudos prévios que evidenciaram que a temática parece não ser suficientemente abordada na formação dos professores (Carvalho et al., 2007; Dias et al., 2013; Gonçalves \& Crenitte, 2014; Lopes \& Crenitte, 2013; Machado et al., 2015; Pereira et al., 2011; Tratis \& Gomes, 2009).

Analisando os itens de menor acerto entre os concluintes, destacou-se o baixo percentual de acertos de conceitos importantes para educadores, por exemplo, o item 20 ("O TDAH é considerado um dos transtornos específicos da aprendizagem") que teve apenas 7\% de acertos, e o item 9 ("Disgrafia e Disortografia são sinônimos e se manifestam da mesma forma"), com $21 \%$ de acerto. A falta de conhecimento desta natureza pode levar à ausência ou inadequação de estratégias pedagógicas específicas, uma vez que são transtornos do neurodesenvolvimento com características e prejuízos diferentes. Por exemplo, um aluno com TDAH pode não ter comprometimento em habilidades acadêmicas e um aluno com TEAp pode não ter comprometimentos comportamentais, como desatenção, hiperatividade e impulsividade.

Os itens que dizem respeito à prevalência do TEAp também tiveram baixo percentual de acertos entre concluintes, como os itens 17 ("O risco relativo de TA é substancialmente maior em parentes de $1^{\circ}$ grau de indivíduos com essas dificuldades"), com $16 \%$ de acerto, e o item 18 ("O TEAp é mais comum no sexo masculino do que no feminino"), com $7 \%$ de acerto. O desconhecimento destes itens pode retardar a adoção de práticas interventivas precoce-preventivas e encaminhamentos para avaliação na idade adequada.

Por fim, os itens 5 ("As dificuldades de aprendizagem oriundas de um TEAp podem ter início tanto durante os anos escolares, como na fase adulta") e 6 ("As dificuldades de aprendizagem oriundas de um TEAp podem ser explicadas a partir do diagnóstico de deficiências intelectuais, acuidade visual ou auditiva não corrigida, outros transtornos mentais ou neurológicos") também tiveram baixo percentual de acerto, respectivamente, $20 \%$ de acerto e $12 \%$ de acerto. Considerando a relevância dos professores na identificação precoce de possíveis sinais compatíveis com perfil de TEAp, preocupa a falta de conhecimento dos concluintes sobre sinais e critérios diagnósticos identificados nos itens 5 e 6 .

Apesar da diferença entre grupos, o escore total de acertos dos concluintes foi baixo, alcançando mediana de 8 num total de 20 questões, ou seja, menos da metade do total de questões do questionário. Esses dados sugerem que, possivelmente, os participantes desta pesquisa podem futuramente apresentar dificuldades para identificar quando seus alunos apresentarem tais dificuldades 
acadêmicas. De fato, outros estudos (Gonçalves \& Crenitte, 2014; Lopes \& Crenitte, 2013; Pereira et al., 2011; Tratis \& Gomes, 2009) verificaram que os professores demonstram ter dificuldades para identificar sinais e características de perfil compatível com um TEAp, além de desconhecerem o tipo de atendimento educacional adequado para estes alunos. Para ampliar o conhecimento dos professores sobre estratégias gerais e específicas para oferecer atendimento educacional adequado aos alunos com TEAp, sugere-se a leitura da "Cartilha da Inclusão Escolar: inclusão baseada em evidências científicas" (Comunidade Aprender Criança, 2014), disponível para download gratuito na internet.

De forma exploratória, foi verificada uma diferença significativa no conhecimento dos alunos entre as instituições. Tal resultado sugere que talvez o assunto não seja abordado de forma uniforme nos cursos de formação de professores. Considerando a relevância do professor na identificação precoce de possíveis sinais compatíveis com perfil de TEAp (Berninger et al., 2008; Padhy et al., 2015), futuros estudos poderiam investigar se há algum parâmetro curricular nacional dos cursos de formação de professores que recomende a inserção de disciplinas que abordem o TEAp.

Ainda de forma exploratória, não houve associação entre o conhecimento que os alunos têm sobre TEAp e outros fatores, tais como idade, renda e situação de trabalho. Estes resultados mostram que os conhecimentos avaliados pelo questionário parecem não estar associados a fatores externos à graduação, como renda ou idade. Entretanto, levanta-se a hipótese que, de fato, o baixo nível de conhecimento verificado na amostra possa estar relacionado à qualidade da formação superior que eles estão recebendo.

\section{CONSIDERAÇÕES FINAIS}

Este estudo possibilitou a construção de um questionário para a avaliação do conhecimento sobre o TEAp com adequadas propriedades psicométricas de precisão e que, no estudo piloto conduzido junto a 178 estudantes de pedagogia, identificou diversas lacunas no conhecimento que eles têm sobre o transtorno. Considerando os resultados positivos encontrados quanto à verificação de propriedades psicométricas do instrumento recomenda-se 0 uso desse questionário (disponível em <https://goo.gl/forms/tDbtUafyy2c5kUn52>), como uma medida que poderá aferir o nível de conhecimentos de estudantes de cursos de pedagogia em relação ao TEAp. A averiguação desse tipo de indicador pode servir como diretriz para o aprimoramento de conteúdos curriculares em cursos de graduação, quando necessário, e, com isso, possibilitar um perfil de formação melhor para futuros professores da educação básica. Essa recomendação está sustentada em um dos achados do estudo, quando verificou-se que os déficits de formação foram semelhantes entre os grupos de ingressantes e concluintes. 
Este trabalho não pretende esgotar a avaliação do conhecimento sobre TEAp de alunos de pedagogia, portanto algumas limitações devem ser consideradas, tais como amostra por conveniência e sem aleatorização de sujeitos, e a ausência de busca por evidências de validade de conteúdo do questionário. Sugere-se que futuros trabalhos possam suprir tais limitações, além de expandir a amostra a fim de ampliar as possibilidades de generalização dos resultados.

Um desdobramento a partir dos achados do estudo é recomendar a inclusão de disciplinas sobre TEAp na grade curricular dos cursos de pedagogia. Esses conhecimentos são fundamentais para a identificação e adoção de estratégias de intervenção apropriadas com os alunos da Educação Básica de escolas onde esses estudantes serão inseridos no mercado de trabalho. Com isso, poderá ser minimizado o impacto deste transtorno na vida dos estudantes.

\section{DECLARAÇÃO DE CONFLITO DE INTERESSES}

Não há conflito de interesses.

\section{REFERÊNCIAS}

American Psychiatric Association - APA (2014). Manual diagnóstico e estatístico de transtorno mentais: DSM- 5. Porto Alegre, RS: Artmed.

Berninger, V. W., Nagy, W., Richards, T., \& Raskind, W. (2008). Developmental dyslexia: A neurolinguistic approach. In G. Rickheit, \& H. Strohner (Eds), Handbook of communication competence (pp. 337-431). Berlin: Walter de Gruyter \& Co.bernin.

Carvalho, F. B., Crenitte, P. A. P., \& Ciasca, S. M. (2007). Distúrbios de aprendizagem na visão do professor. Revista Psicopedagogia, 24(75), 22939.

Comunidade Aprender Criança (2014). Cartilha da Inclusão Escolar: Inclusão baseada em evidências científicas. Ribeirão Preto, SP: Ed. Instituto Glia.

de Wit, E., van Dijk, P., Hanekamp, S., Visser-Bochane, M., Steenbergen, B., van der Schans, C. P., \& Luinge, M. R. (2018). Same or different: The overlap between children with auditory processing disorders and children with other developmental disorders: A systematic review. Ear and Hearing, 39(1), 119. doi:10.1097/AUD.0000000000000479.

Dias, M. de A. H., Pereira, M. M. de B., \& Van Borsel, J. (2013). Avaliação do conhecimento sobre a discalculia entre educadores. Audiology Communication Research, 18(2), 93-100. doi:10.1590/S231764312013000200007. 
Fernandes, G. B., \& Crenitte, P. A. P. (2008). O conhecimento dos professores de $1^{\mathrm{a}}$ a $4^{\mathrm{a}}$ série quanto aos distúrbios de leitura e escrita. Revista CEFAC. 10(2), 182-90. doi:10.1590/S1516-18462008000200007.

Flatters, I., Hill, L.J., Williams, J. H., Barber, S. E., \& Mon-Williams, M. (2014). Manual control age and sex differences in 4 to 11 year old children. PLoSOne, 9(2):e88692. doi:10.1371/journal.pone.0088692.

Fortes, I. S., Paula, C. S., Oliveira, M. C., Bordin, I. A., de Jesus Mari, J., \& Rohde, L.A. (2016). A cross-sectional study to assess the prevalence of DSM-5 specific learning disorders in representative school samples from the second to sixth grade in Brazil. European Child \& Adolescent Psychiatry, 25(2),195-207. doi:10.1007/s00787-015-0708-2.

George, D., \& Mallery, P. (2003). SPSS for Windows step by step: A simple guide and reference, 11.0 update, 4th ed. Boston: Allyn \& Bacon.

Gonçalves, T. S. (2011) Desenvolvimento de material educacional interativo para orientação de professores do ensino fundamental quanto aos Distúrbios da Linguagem Escrita (Dissertação de mestrado). Universidade de São Paulo, Bauru, Brasil.

Gonçalves, T. S., \& Crenitte, P. A. P. (2014). Concepções de professoras de ensino fundamental sobre os transtornos de aprendizagem. Revista CEFAC, 16(3), 817-829. doi:10.1590/1982-0216201427312.

Kamala, R., \& Ramganesh, E. (2013). Knowledge of specific learning disabilities among teacher educators in Puducherry, Union Territory in India. International Review of Social Sciences and Humanities, 6, 168-75.

Lingeswaran, A. (2013). Assessing knowledge of primary school teachers on specific learning disabilities in two schools in India. Journal of Education and Health Promotion, 2, 30. doi:10.4103/2277-9531.115807.

Lopes, R. C. F., \& Crenitte, P. A. P. (2013). Estudo analítico do conhecimento do professor a respeito dos distúrbios de aprendizagem. Revista CEFAC, 15(5), 1214-1226. doi:10.1590/S1516-18462012005000091.

Machado, A. C., Borges, K. K., Costa, M. P. R. C., \& Almeida, M. A. (2015). Perfil dos professores do ensino fundamental de uma rede pública sobre transtornos de aprendizagem. Revista Educação Especial, 28(52), 417-428. doi: $10.5902 / 1984686 \times 7907$.

Osborn, E., \& Pereira, L. D. (2012). Neuropsychological aspects of 10-year-old children. Einstein, 10(4), 433-438. doi:10.1590/S167945082012000400007.

Padhy, S. K., Goel, S., Das, S. S., Sarkar, S., Sharma, V., \& Panigrahi, M. (2015). Perceptions of teachers about learning disorder in a northern city of India. Journal of Family Medicine and Primary Care, 4(3), 432-434. doi: $10.4103 / 2249-4863.161347$. 
Pereira, L. V., Simões, M. G., Siqueira, C. M., \& Alves, L. M. (2011). Estudo investigativo sobre o conhecimento da dislexia em educadores da rede pública e privada dos municípios de Belo Horizonte e de Nova Lima. Revista Tecer. Belo Horizonte, 4(6). doi:10.15601/1983-7631/rt.v4n6p26-40.

Silva, T, A., \& Cavalcante, L. I. C. (2015). Habilidades sociais e características pessoais em escolares de Belém. Psicologia: Reflexão e Crítica, 28(4), 850858. doi: 10.1590/1678-7153.201528424.

Soriano-Ferrer, M., Echegaray-Bengoa, J., \& Joshi, M. R. (2016). Knowledge and beliefs about developmental dyslexia, in pre-service and in-service Spanishspeaking teachers. Annals of Dyslexia, 66, 91-110. doi:10.1007/s11881015-0111-1.

Tratis, S. A., \& Gomes, T. S. (2009). Nível de conhecimento dos professores de Guarapuava - PR sobre a dislexia do desenvolvimento. Anais da SIEPE Semana de Integração Ensino, Pesquisa e Extensão, s/n.

Washburn, E. K., Cantrell, E. S. B., \& Joshi., R. M. (2013). What do preservice teachers from the USA and the UK know about dyslexia? Dyslexia, 20(1), 118. doi: $10.1002 /$ dys. 1459 . 
Sobre os autores

Camila Barbosa Riccardi León é graduada em Pedagogia pelo Centro Universitário Claretiano e em Letras pela Universidade do Vale do Paraíba, pós-graduada em Língua Inglesa pela Universidade de Taubaté, pós-graduada em Psicopedagogia pela Universidade Presbiteriana Mackenzie, mestre e doutora em Distúrbios do Desenvolvimento pela Universidade Presbiteriana Mackenzie.

camilaleon30@gmail.com

Ronê Paiano é graduado em educação física pela Universidade do $A B C$, mestre em Educação Arte e História da Cultura e doutor em Distúrbios do Desenvolvimento pela Universidade Presbiteriana Mackenzie. rone.pefe@gmail.com

Analice Oliveira Fragoso é pedagoga pela Universidade São Marcos, especialista em Psicopedagogia, mestre e doutora em Distúrbios do Desenvolvimento pela Universidade Presbiteriana Mackenzie. analicefragoso@hotmail.com Alisson Rogério Caetano de Siqueira é psicólogo pelo Centro Universitário do Norte, teólogo pelo Seminário Presbiteriano do Norte, mestre e doutor em Distúrbios do Desenvolvimento pela Universidade Presbiteriana Mackenzie. É professor da Fatesul-IPB e FAEC-INESUL. psicoalisson@hotmail.com Maria Cristina Triguero Veloz Teixeira é psicóloga pela Universidade de Havana, Cuba, mestre em Psicologia pela Universidade Federal de Santa Catarina e Doutora em Saúde pela Universidade Federal de Santa Catarina, bolsista Produtividade do CNPq-1D. É docente do Programa de Pós-graduação em Distúrbios do Desenvolvimento e Coordenadora Geral da Pós-Graduação Stricto Sensu da Universidade Presbiteriana Mackenzie. mcris@mackenzie.br Alessandra Gotuzo Seabra é psicóloga, Mestre, Doutora e com Pós-doutorado em Psicologia pela Universidade de São Paulo. Bolsista Produtividade do CNPq- 1B. É docente e coordenadora do Programa de Pós-graduação em Distúrbios do Desenvolvimento da Universidade Presbiteriana Mackenzie. alessandra.seabra@mackenzie.br

A contribuição de cada autor pode ser atribuída como se segue: C.B.R.L., R.P., A.O.F., A.R.C.S., M.C.T.V.T. e A.G.S. contribuíram para a conceitualização, investigação e visualização do artigo; A.O.F. e A.R.C.S. fizeram a redação inicial do artigo (rascunho) e C.B.R.L., R.P., M.C.T.V.T. e A.G.S. são os responsáveis pela redação final (revisão e edição).

Recebido em: 29/11/2017

$1^{\text {a }}$ revisão em: 23/03/2018

Aceito em: 26/05/2018 